\title{
INDEX TRANSFORMS WITH THE SQUARE OF BESSEL FUNCTIONS
}

\author{
S. YAKUBOVICH
}

\begin{abstract}
New index transforms, involving the square of Bessel functions of the first kind as the kernel are considered. Mapping properties such as the boundedness and invertibility are investigated for these operators in the Lebesgue spaces. Inversion theorems are proved. As an interesting application, a solution to the initial value problem for the third order partial differential equation, involving the Laplacian, is obtained.
\end{abstract}

\section{INTRODUCTION AND PRELIMINARY RESULTS}

Let $f(x), g(\tau), x \in \mathbb{R}_{+}, \tau \in \mathbb{R}$ be complex -valued functions. The main goal of this paper is to investigate mapping properties of the following index transforms [1], involving the square of Bessel's function of the first kind in the kernel, namely,

$$
\begin{array}{ll}
(F f)(\tau)=\frac{\sqrt{\pi}}{\cosh (\pi \tau)} \int_{0}^{\infty} \operatorname{Re}\left[J_{i \tau}^{2}(\sqrt{x})\right] f(x) d x, & \tau \in \mathbb{R}, \\
(G g)(x)=\sqrt{\pi} \int_{-\infty}^{\infty} \operatorname{Re}\left[J_{i \tau}^{2}(\sqrt{x})\right] \frac{g(\tau) d \tau}{\cosh (\pi \tau)}, \quad x \in \mathbb{R}_{+},
\end{array}
$$

where $i$ is the imaginary unit and Re denotes the real part of a complex -valued function. Bessel's function of the first kind $J_{v}(z)$ [2], Vol. II satisfies the differential equation

$$
z^{2} \frac{d^{2} u}{d z^{2}}+z \frac{d u}{d z}+\left(z^{2}-v^{2}\right) u=0 .
$$

It has the asymptotic behavior

$$
\begin{gathered}
J_{v}(z)=\sqrt{\frac{2}{\pi z}} \cos \left(z-\frac{\pi}{4}(2 v+1)\right)[1+O(1 / z)], z \rightarrow \infty,-\frac{\pi}{2}<\arg z<\frac{3 \pi}{2}, \\
J_{v}(z)=O\left(z^{v}\right), z \rightarrow 0 .
\end{gathered}
$$

and the following series expansion

$$
J_{v}(z)=\sum_{k=0}^{\infty} \frac{(-1)^{k}(z / 2)^{2 k+v}}{k ! \Gamma(k+v+1)}, z, v \in \mathbb{C}
$$

where $\Gamma(z)$ is Euler's gamma function [2], Vol. I. Using the reduction formula for the gamma function [2], Vol. I we find for $\operatorname{Re} v \geq 0$

$$
|\Gamma(k+v+1)|=|\Gamma(v+1)(1+v)(2+v) \ldots(k+v)| \geq k !|\Gamma(v+1)| .
$$

Date: October 20, 2015.

2000 Mathematics Subject Classification. 44A15, 33C10, 44A05 .

Key words and phrases. Index Transforms, Bessel functions, Fourier transform, Mellin transform, Initial value problem. 
Hence we derive from (1.6)

$$
\begin{gathered}
\left|J_{v}(z)\right| \leq e^{-\operatorname{Im} v \arg z} \sum_{k=0}^{\infty} \frac{(|z| / 2)^{2 k+\operatorname{Re} v}}{k !|\Gamma(k+v+1)|} \leq e^{-\operatorname{Im} v \arg z} \frac{(|z| / 2)^{\operatorname{Re} v}}{|\Gamma(v+1)|} \sum_{k=0}^{\infty} \frac{(|z| / 2)^{2 k}}{(k !)^{2}} \\
\leq e^{|z|-\operatorname{Im} v \arg z} \frac{(|z| / 2)^{\operatorname{Re} v}}{|\Gamma(v+1)|},
\end{gathered}
$$

coming up to the following inequality for the Bessel function of the first kind

$$
\left|J_{v}(z)\right| \leq\left(\frac{|z|}{2}\right)^{\operatorname{Re} v} \frac{e^{|z|-\operatorname{Im} v \arg z}}{|\Gamma(v+1)|}, z, v \in \mathbb{C} .
$$

Taking into account the value

inequality (1.7) takes the form

$$
|\Gamma(1+i \tau)|=\sqrt{\frac{\pi \tau}{\sinh (\pi \tau)}}
$$

$$
\left|J_{i \tau}(x)\right| \leq e^{x} \sqrt{\frac{\sinh (\pi \tau)}{\pi \tau}}, x>0, \tau \in \mathbb{R} .
$$

In the meantime, appealing to relation (8.4.19.17) in [4], Vol. III, we find the following Mellin-Barnes integral representation for the kernel

$$
\Phi_{\tau}(x)=\frac{\sqrt{\pi}}{\cosh (\pi \tau)} \operatorname{Re}\left[J_{i \tau}^{2}(\sqrt{x})\right]
$$

in (1.1), (1.2), namely,

$$
\Phi_{\tau}(x)=\frac{1}{2 \pi i} \int_{\gamma-i \infty}^{\gamma+i \infty} \frac{\Gamma(s+i \tau) \Gamma(s-i \tau) \Gamma(1 / 2-s)}{\Gamma(s) \Gamma(1-s) \Gamma(1-s)} x^{-s} d s, x>0,
$$

where $\gamma$ is taken from the interval $(0,1 / 4)$. It guarantees the absolute convergence of the integral (1.9) since appealing to the Stirling asymptotic formula for the gamma- function [2], Vol. I

$$
\frac{\Gamma(s+i \tau) \Gamma(s-i \tau) \Gamma(1 / 2-s)}{\Gamma(s) \Gamma(1-s) \Gamma(1-s)}=O\left(|s|^{2 \gamma-3 / 2}\right),|s| \rightarrow \infty .
$$

Integral (1.9) can be involved to represent $\Phi_{\tau}(x)$ for all $x>0, \tau \in \mathbb{R}$ as the Fourier cosine transform [5]. Indeed, we have

Lemma 1. Let $x>0, \tau \in \mathbb{R}$. Then $\Phi_{\tau}(x)$ has the representation in terms of the Fourier cosine transform

$$
\Phi_{\tau}(x)=-\frac{1}{\sqrt{\pi}} \frac{\partial}{\partial x} \int_{0}^{\infty} \frac{\sqrt{x} \cos (\tau u)}{\cosh (u / 2)} \mathbf{L}_{1}\left(2 i \sqrt{x} \cosh \left(\frac{u}{2}\right)\right) d u,
$$

where $\mathbf{L}_{1}(z)$ is the modified Struve function of the index one [2], Vol. II.

Proof. Integrating in (1.9) with respect to $x$, we change the order of integration in the right-hand side of the obtained equality by virtue of the absolute convergence of the iterated integral. As a result we obtain

$$
\frac{1}{x} \int_{0}^{x} \Phi_{\tau}(y) d y=\frac{1}{2 \pi i} \int_{\gamma-i \infty}^{\gamma+i \infty} \frac{\Gamma(s+i \tau) \Gamma(s-i \tau) \Gamma(1 / 2-s)}{\Gamma(s) \Gamma(1-s) \Gamma(2-s)} x^{-s} d s, x>0,
$$

Hence, appealing to the reciprocal formulae via the Fourier cosine transform (cf. formula (1.104) in [1])

$$
\int_{0}^{\infty} \Gamma(s+i \tau) \Gamma(s-i \tau) \cos (\tau y) d \tau=\frac{\pi}{2^{2 s}} \frac{\Gamma(2 s)}{\cosh ^{2 s}(y / 2)}, \operatorname{Re} s>0,
$$




$$
\Gamma(s+i \tau) \Gamma(s-i \tau)=\frac{\Gamma(2 s)}{2^{2 s-1}} \int_{0}^{\infty} \frac{\cos (\tau y)}{\cosh ^{2 s}(y / 2)} d y,
$$

we replace the gamma-product $\Gamma(s+i \tau) \Gamma(s-i \tau)$ in the integral (1.12) by its integral representation (1.14) and change the order of integration via the absolute convergence, which can be justified using the Stirling asymptotic formula for the gamma-function. Then, employing the duplication formula for the gammafunction [2], Vol. I, we derive

$$
\begin{gathered}
\frac{1}{x} \int_{0}^{x} \Phi_{\tau}(y) d y=\frac{1}{2 \pi \sqrt{\pi} i} \int_{0}^{\infty} \cos (\tau u) \int_{\gamma-i \infty}^{\gamma+i \infty} \frac{\Gamma(s+1 / 2) \Gamma(1 / 2-s)}{\Gamma(1-s) \Gamma(2-s)}\left(x \cosh ^{2}(u / 2)\right)^{-s} d s d u \\
=-\frac{1}{\sqrt{\pi x}} \int_{0}^{\infty} \frac{\cos (\tau u)}{\cosh (u / 2)} \mathbf{L}_{1}\left(2 i \sqrt{x} \cosh \left(\frac{u}{2}\right)\right) d y
\end{gathered}
$$

where the inner integral with respect to $s$ is calculated via Slater's theorem and relation (7.14.2.79) in [4], Vol. III. Hence after the differentiation with respect to $x$ we arrive at (1.11), completing the proof of Lemma 1 .

Further, recalling the Mellin-Barnes representation (1.9) of the kernel $\Phi_{\tau}(x)$, we will derive an ordinary differential equation whose particular solution is $\Phi_{\tau}(x)$. Precisely, it is given by

Lemma 2. For each $\tau \in \mathbb{R}$ the function

$$
\Phi_{\tau}(x)=\frac{\sqrt{\pi}}{\cosh (\pi \tau)} \operatorname{Re}\left[J_{i \tau}^{2}(\sqrt{x})\right]
$$

is a fundamental solution of the following third order differential equation with variable coefficients

$$
x^{2} \frac{d^{3} \Phi_{\tau}}{d x^{3}}+3 x \frac{d^{2} \Phi_{\tau}}{d x^{2}}+\left(\tau^{2}+x-7\right) \frac{d \Phi_{\tau}}{d x}+\frac{\Phi_{\tau}}{2}=0, x>0 .
$$

Proof. The asymptotic behavior (1.10) of the integrand in (1.9) and the absolute and uniform convergence of the integral allow to integrate (1.9) twice with respect to $x$. Hence making use the reduction formula for the gamma- function [2], Vol. I we obtain

$$
\int_{0}^{x} d u \int_{0}^{u} \Phi_{\tau}(v) d v=\frac{1}{2 \pi i} \int_{\gamma-i \infty}^{\gamma+i \infty} \frac{\Gamma(s+i \tau) \Gamma(s-i \tau) \Gamma(1 / 2-s)}{\Gamma(s) \Gamma(1-s) \Gamma(3-s)} x^{2-s} d s .
$$

Then, plainly, with the reduction formula for the gamma-function and simple changes of variables we get

$$
\begin{gathered}
\left(x \frac{d}{d x}\right)^{2}\left[\frac{1}{x^{2}} \int_{0}^{x} d u \int_{0}^{u} \Phi_{\tau}(v) d v\right]=\frac{1}{2 \pi i} \int_{\gamma-i \infty}^{\gamma+i \infty} \frac{s^{2} \Gamma(s+i \tau) \Gamma(s-i \tau) \Gamma(1 / 2-s)}{\Gamma(s) \Gamma(1-s) \Gamma(3-s)} x^{-s} d s \\
=-\frac{\tau^{2}}{x^{2}} \int_{0}^{x} d u \int_{0}^{u} \Phi_{\tau}(v) d v+\frac{1}{2 \pi i} \int_{\gamma-i \infty}^{\gamma+i \infty} \frac{\Gamma(1+s+i \tau) \Gamma(1+s-i \tau) \Gamma(1 / 2-s)}{\Gamma(s) \Gamma(1-s) \Gamma(3-s)} x^{-s} d s \\
=-\frac{\tau^{2}}{x^{2}} \int_{0}^{x} d u \int_{0}^{u} \Phi_{\tau}(v) d v-\frac{1}{2 \pi i} \int_{1+\gamma-i \infty}^{1+\gamma+i \infty} \frac{\Gamma(s+i \tau) \Gamma(s-i \tau) \Gamma(1 / 2-s)(1 / 2-s)}{\Gamma(s) \Gamma(1-s) \Gamma(1-s)(3-s)(2-s)(1-s)} x^{1-s} d s .
\end{gathered}
$$

Hence after multiplication by $x^{2}$, we differentiate three times the obtained equality and use (1.9) to find

$$
\begin{gathered}
\frac{d^{3}}{d x^{3}}\left[x^{2}\left(x \frac{d}{d x}\right)^{2}\left[\frac{1}{x^{2}} \int_{0}^{x} d u \int_{0}^{u} \Phi_{\tau}(v) d v\right]\right]=-\tau^{2} \frac{d \Phi_{\tau}}{d x}-\sqrt{x} \frac{d}{d x}\left(\sqrt{x} \Phi_{\tau}(x)\right) \\
=-\left(\tau^{2}+x\right) \frac{d \Phi_{\tau}}{d x}-\frac{\Phi_{\tau}}{2}
\end{gathered}
$$


Meanwhile, the left-hand side of (1.17) can be simplified as follows

$$
\begin{gathered}
\frac{d^{3}}{d x^{3}}\left[x^{2}\left(x \frac{d}{d x}\right)^{2}\left[\frac{1}{x^{2}} \int_{0}^{x} d u \int_{0}^{u} \Phi_{\tau}(v) d v\right]\right]=\frac{d^{3}}{d x^{3}}\left[x^{3} \frac{d}{d x}\left[-\frac{2}{x^{2}} \int_{0}^{x} d u \int_{0}^{u} \Phi_{\tau}(v) d v+\frac{1}{x} \int_{0}^{x} \Phi_{\tau}(v) d v\right]\right] \\
=\frac{d^{3}}{d x^{3}}\left[-4 \int_{0}^{x} d u \int_{0}^{u} \Phi_{\tau}(v) d v-3 x \int_{0}^{x} \Phi_{\tau}(v) d v+x^{2} \Phi_{\tau}\right]=x^{2} \frac{d^{3} \Phi_{\tau}}{d x^{3}}+3 x \frac{d^{2} \Phi_{\tau}}{d x^{2}}-7 \frac{d \Phi_{\tau}}{d x} .
\end{gathered}
$$

Finally, combining with (1.17), we arrive at the equation (1.15), completing the proof of Lemma 2.

\section{BOUNDEDNESS AND INVERSION PROPERTIES OF THE INDEX TRANSFORM (1.1)}

In order to investigate the mapping properties of the index transform (1.1) we will use the Mellin transform technique developed in [3]. Precisely, the Mellin transform is defined, for instance, in $L_{v, p}\left(\mathbb{R}_{+}\right), 1<$ $p \leq 2$ (see details in [5]) by the integral

$$
f^{*}(s)=\int_{0}^{\infty} f(x) x^{s-1} d x
$$

being convergent in mean with respect to the norm in $L_{q}(v-i \infty, v+i \infty), q=p /(p-1)$. Moreover, the Parseval equality holds for $f \in L_{v, p}\left(\mathbb{R}_{+}\right), g \in L_{1-v, q}\left(\mathbb{R}_{+}\right)$

$$
\int_{0}^{\infty} f(x) g(x) d x=\frac{1}{2 \pi i} \int_{v-i \infty}^{v+i \infty} f^{*}(s) g^{*}(1-s) d s .
$$

The inverse Mellin transform is given accordingly

$$
f(x)=\frac{1}{2 \pi i} \int_{v-i \infty}^{v+i \infty} f^{*}(s) x^{-s} d s,
$$

where the integral converges in mean with respect to the norm in $L_{v, p}\left(\mathbb{R}_{+}\right)$

$$
\|f\|_{v, p}=\left(\int_{0}^{\infty}|f(x)|^{p} x^{v p-1} d x\right)^{1 / p} .
$$

In particular, letting $v=1 / p$ we get the usual space $L_{1}\left(\mathbb{R}_{+}\right)$. Further, denoting by $C\left(\mathbb{R}_{+}\right)$the space of bounded continuous functions, we have

Theorem 1. The index transform (1.1) is well-defined as a bounded operator $F: L_{1}\left(\mathbb{R}_{+} ; e^{2 \sqrt{x}} d x\right) \rightarrow$ $C(\mathbb{R})$ and the following norm inequality takes place

$$
\left\|\left.F f\right|_{C(\mathbb{R})} \equiv \sup _{\tau \in \mathbb{R}}|(F f)(\tau)| \leq \sqrt{\pi}|| f\right\|_{L_{1}\left(\mathbb{R}_{+} ; e^{2 \sqrt{x}} d x\right)} .
$$

Moreover, let the Mellin transform (2.1) of $f$ satisfy the condition

$$
\frac{f^{*}(s)}{\Gamma(s) \Gamma(1-s)} \in L_{q}(1-v-i \infty, 1-v+i \infty),
$$

where

$$
0<v<\frac{3}{4}-\frac{1}{2 p}, 1<p<2, \frac{1}{p}+\frac{1}{q}=1
$$

Then

$$
(F f)(\tau)=\frac{\sqrt{\pi}}{\cosh (\pi \tau)} \int_{0}^{\infty} \operatorname{Re}\left[I_{i \tau}\left(\frac{x}{2}\right)\right] e^{-x / 2} \varphi(x) d x,
$$


where $I_{\mu}(z)$ is the modified Bessel function of the first kind,

$$
\varphi(x)=\frac{1}{2 \pi i} \int_{1-v-i \infty}^{1-v+i \infty} \frac{f^{*}(s)}{\Gamma(s)} x^{-s} d s,
$$

and integral (2.9) converges with respect to the norm in $L_{1-v, p}\left(\mathbb{R}_{+}\right)$.

Proof. The proof of the norm inequality (2.5) is straightforward from (1.1) and inequality (1.8). The continuity of $(F f)(\tau)$ follows from the absolute and uniform convergence of the corresponding integral. In fact, we derive

$$
\begin{gathered}
|(F f)(\tau)| \leq \frac{\sqrt{\pi}}{\cosh (\pi \tau)} \int_{0}^{\infty}\left|J_{i \tau}(\sqrt{x})\right|^{2}|f(x)| d x \\
\leq \sqrt{\pi} \frac{\tanh (\pi \tau)}{\pi \tau} \int_{0}^{\infty}|f(x)| e^{2 \sqrt{x}} d x \leq\left.\sqrt{\pi}|| f\right|_{L_{1}\left(\mathbb{R}_{+} ; e^{2 \sqrt{x}} d x\right)} .
\end{gathered}
$$

This proves (2.5). Next, $f^{*}(1-s) \in L_{q}(v-i \infty, v+i \infty)$ via condition (2.6). Indeed, we have

$$
\begin{gathered}
\int_{v-i \infty}^{v+i \infty}\left|f^{*}(1-s)\right|^{q}|d s|=\int_{1-v-i \infty}^{1-v+i \infty}\left|\Gamma(s) \Gamma(1-s) f^{*}(s)\right|^{q} \frac{|d s|}{|\Gamma(s) \Gamma(1-s)|^{q}} \\
\leq[\Gamma(v) \Gamma(1-v)]^{q} \int_{1-v-i \infty}^{1-v+i \infty}\left|f^{*}(s)\right|^{q} \frac{|d s|}{|\Gamma(s) \Gamma(1-s)|^{q}}<\infty .
\end{gathered}
$$

Therefore, via Theorem 86 in [5] we see that $f \in L_{1-v, p}\left(\mathbb{R}_{+}\right)$. Meanwhile, the asymptotic behavior (1.10) of the integrand in (1.9) guarantees that it belongs to the space $L_{p}(v-i \infty, v+i \infty)$ with $v$, satisfying condition (2.7). Consequently, (1.9) and the Parseval identity (2.2) lead to the following representation

$$
(F f)(\tau)=\frac{1}{2 \pi i} \int_{v-i \infty}^{v+i \infty} \frac{\Gamma(s+i \tau) \Gamma(s-i \tau) \Gamma(1 / 2-s)}{\Gamma(s) \Gamma^{2}(1-s)} f^{*}(1-s) x^{-s} d s .
$$

Analogously to (2.10) we show that $f^{*}(1-s) / \Gamma(1-s) \in L_{q}(v-i \infty, v+i \infty)$ and hence (see (2.9)) $\varphi \in$ $L_{1-v, p}\left(\mathbb{R}_{+}\right)$. But from the asymptotic behavior of the modified Bessel function of the first kind (see [2], Vol. II) we find

$$
\begin{gathered}
e^{-x / 2} \operatorname{Re}\left[I_{i \tau}\left(\frac{x}{2}\right)\right]=O(1), x \rightarrow 0, \\
e^{-x / 2} \operatorname{Re}\left[I_{i \tau}\left(\frac{x}{2}\right)\right]=O\left(\frac{1}{\sqrt{x}}\right), x \rightarrow \infty .
\end{gathered}
$$

Therefore, the kernel of (2.8) belongs to $L_{v, q}\left(\mathbb{R}_{+}\right)$and integral converges absolutely. Moreover, Lemma 1 in [7], the Parseval identity (2.2) and (2.11) establish equality (2.8), completing the proof of Theorem 1.

The inversion formula for the transform (1.1) is given by

Theorem 2. Under conditions of Theorem 1 let also the Mellin transform $f^{*}(s)$ be analytic in the strip

$$
\frac{1}{2 p}-\frac{1}{4}<\operatorname{Re} s=1-v<\frac{7}{4}-\frac{1}{2 p}, 1<p<2
$$

and

$$
\frac{f^{*}(s)}{\Gamma(s) \Gamma(1-s)} \in L_{q}(1-v-i \infty, 1-v+i \infty) \cap L_{1}(1-v-i \infty, 1-v+i \infty),|v|<\frac{3}{4}-\frac{1}{2 p} .
$$

Then, assuming that the index transform (1.1) satisfies the integrability condition $(F f)(\tau) \in L_{1}\left(\mathbb{R}_{+} ; \tau e^{\pi \tau} d \tau\right)$, it has the following inversion formula for all $x>0$ 


$$
\begin{gathered}
f(x)=2 \sqrt{\pi} \frac{d}{d x} \int_{0}^{\infty} \tau \operatorname{coth}(\pi \tau)\left[\frac{\operatorname{Im}\left[J_{i \tau}^{2}(\sqrt{x})\right]}{\sinh (\pi \tau)}-\frac{1}{\pi}\left[\left.J_{i \tau}(\sqrt{x}) \frac{\partial J_{\varepsilon-i \tau}(\sqrt{x})}{\partial \varepsilon}\right|_{\varepsilon=0}\right.\right. \\
\left.\left.+\left.J_{-i \tau}(\sqrt{x}) \frac{\partial J_{\varepsilon+i \tau}(\sqrt{x})}{\partial \varepsilon}\right|_{\varepsilon=0}\right]\right](F f)(\tau) d \tau,
\end{gathered}
$$

where $\operatorname{Im}$ denotes the imaginary part of a complex -valued function and the corresponding integral converges absolutely.

Proof. Following the same scheme as in [7], Th. 2, we verify conditions of the Lebedev expansion theorem for the index transform with the square of the Macdonald function as the kernel $K_{i \tau}^{2}(\sqrt{x})$ [2], Vol. II, coming up to the equality

$$
\int_{x}^{\infty} \frac{1}{2 \pi i} \int_{1-v-i \infty}^{1-v+i \infty} \frac{f^{*}(s) y^{-s}}{\Gamma(s) \Gamma(1-s)} d s d y=\frac{2}{\pi^{2} \sqrt{\pi}} \int_{0}^{\infty} \tau \sinh (2 \pi \tau) K_{i \tau}^{2}(\sqrt{x})(F f)(\tau) d \tau, x>0 .
$$

Fulfilling the integration in the left-hand side of (2.14) when $v \in(-3 / 4+1 /(2 p), 0)$, it becomes

$$
-\frac{1}{2 \pi i} \int_{1-v-i \infty}^{1-v+i \infty} \frac{f^{*}(s) x^{1-s}}{\Gamma(s) \Gamma(2-s)} d s=\frac{2}{\pi^{2} \sqrt{\pi}} \int_{0}^{\infty} \tau \sinh (2 \pi \tau) K_{i \tau}^{2}(\sqrt{x})(F f)(\tau) d \tau .
$$

Hence

$$
\begin{gathered}
-\frac{1}{2 \pi i} \int_{0}^{\infty} \frac{d y}{(x+y)^{2}} \int_{1-v-i \infty}^{1-v+i \infty} \frac{f^{*}(s) y^{1-s}}{\Gamma(s) \Gamma(2-s)} d s \\
=\frac{2}{\pi^{2} \sqrt{\pi}} \int_{0}^{\infty} \frac{d y}{(x+y)^{2}} \int_{0}^{\infty} \tau \sinh (2 \pi \tau) K_{i \tau}^{2}(\sqrt{y})(F f)(\tau) d \tau .
\end{gathered}
$$

The interchange of the order of integration in both sides of the latter equality is allowed due to Fubini's theorem under imposed conditions. Thus, taking into account the value of the elementary beta-integral and the inverse Mellin transform (2.3), we end up with the inversion formula of the index transform (1.1), namely

$$
f(x)=\frac{2}{\pi^{2} \sqrt{\pi}} \int_{0}^{\infty} \tau \sinh (2 \pi \tau) K(x, \tau)(F f)(\tau) d \tau, x>0,
$$

where

$$
K(x, \tau)=-\int_{0}^{\infty} \frac{K_{i \tau}^{2}(\sqrt{y}) d y}{(x+y)^{2}} .
$$

In the meantime integral (2.17), which is absent in the literature, can be calculated in the following way. Using relations (8.4.23.27) and (8.4.2.5) in [4], Vol. III and the Parseval equality (2.2), we write

$$
-\int_{0}^{\infty} \frac{K_{i \tau}^{2}(\sqrt{y}) d y}{(x+y)^{2}}=\frac{\sqrt{\pi}}{4 \pi i} \frac{d}{d x} \int_{\gamma-i \infty}^{\gamma+i \infty} \Gamma(s+i \tau) \Gamma(s-i \tau) \Gamma^{2}(s) \Gamma(1-s) \frac{x^{-s}}{\Gamma(1 / 2+s)} d s,
$$

where $0<\gamma<1$. On the other hand, the Lebedev inequality for the Macdonald function (cf. [3], p. 99)

$$
\left|K_{i \tau}(x)\right| \leq \frac{x^{-1 / 4}}{\sqrt{\sinh (\pi \tau)}}, x, \tau>0
$$

and the estimate

$$
\int_{0}^{\infty} \tau \sinh (2 \pi \tau)|K(x, \tau)(F f)(\tau)| d \tau \leq 2 \int_{0}^{\infty} \tau \cosh (2 \pi \tau)|(F f)(\tau)| d \tau \int_{0}^{\infty} \frac{y^{-1 / 4} d y}{(x+y)^{2}}<\infty, x>0
$$


under condition $(F f)(\tau) \in L_{1}\left(\mathbb{R}_{+} ; \tau e^{\pi \tau} d \tau\right)$ permit us to write (2.16) in the form

$$
f(x)=\frac{2}{\pi^{2} \sqrt{\pi}} \frac{d}{d x} \int_{0}^{\infty} \int_{0}^{\infty} \tau \sinh (2 \pi \tau) K_{i \tau}^{2}(\sqrt{y})(F f)(\tau) \frac{d y d \tau}{x+y},
$$

where via the uniform convergence and (2.18)

$$
\begin{aligned}
& \int_{0}^{\infty} \frac{K_{i \tau}^{2}(\sqrt{y}) d y}{x+y}=\frac{\sqrt{\pi}}{4 \pi i} \int_{\gamma-i \infty}^{\gamma+i \infty} \Gamma(s+i \tau) \Gamma(s-i \tau) \Gamma^{2}(s) \Gamma(1-s) \frac{x^{-s}}{\Gamma(1 / 2+s)} d s \\
& =\frac{\sqrt{\pi}}{4 \pi i} \lim _{\varepsilon \rightarrow 0} \int_{\gamma-i \infty}^{\gamma+i \infty} \Gamma(s+i \tau) \Gamma(s-i \tau) \Gamma(s+\varepsilon) \Gamma(s-\varepsilon) \Gamma(1-s) \frac{x^{-s}}{\Gamma(1 / 2+s)} d s .
\end{aligned}
$$

Hence the Slater theorem is applied, involving simple left-hand poles $s= \pm i \tau-n, s= \pm \varepsilon-n, n \in \mathbb{N}_{0}$. Calculating the corresponding residues and then passing to the limit when $\varepsilon \rightarrow 0$ with the use of relation (7.15.1.3) in [4], Vol. III, we find the result

$$
\begin{gathered}
\frac{1}{2 \pi i} \lim _{\varepsilon \rightarrow 0} \int_{\gamma-i \infty}^{\gamma+i \infty} \Gamma(s+i \tau) \Gamma(s-i \tau) \Gamma(s+\varepsilon) \Gamma(s-\varepsilon) \Gamma(1-s) \frac{x^{-s}}{\Gamma(1 / 2+s)} d s \\
=\pi^{2} \sqrt{\pi}\left[\frac{\operatorname{Im}\left[J_{i \tau}^{2}(\sqrt{x})\right]}{\sinh ^{3}(\pi \tau)}+\lim _{\varepsilon \rightarrow 0} \frac{J_{-\varepsilon-i \tau}(\sqrt{x}) J_{-\varepsilon+i \tau}(\sqrt{x})-J_{\varepsilon-i \tau}(\sqrt{x}) J_{\varepsilon+i \tau}(\sqrt{x})}{\sin (\pi \varepsilon)(\cosh (2 \pi \tau)-\cos (2 \pi \varepsilon))}\right] \\
=\frac{\pi^{2} \sqrt{\pi}}{\sinh ^{2}(\pi \tau)}\left[\frac{\operatorname{Im}\left[J_{i \tau}^{2}(\sqrt{x})\right]}{\sinh (\pi \tau)}-\frac{1}{\pi}\left[\left.J_{i \tau}(\sqrt{x}) \frac{\partial J_{\varepsilon-i \tau}(\sqrt{x})}{\partial \varepsilon}\right|_{\varepsilon=0}+\left.J_{-i \tau}(\sqrt{x}) \frac{\partial J_{\varepsilon+i \tau}(\sqrt{x})}{\partial \varepsilon}\right|_{\varepsilon=0}\right] .\right.
\end{gathered}
$$

Therefore, combining with (2.20), (2.21), we arrive at the inversion formula (2.13), completing the proof of Theorem 2 .

\section{THE INDEX TRANSFORM (1.2)}

The boundedness and invertibility properties for the index transform (1.2) will be examined below. We begin with

Theorem 3. The $g \in L_{1}(\mathbb{R})$. Then $(G g)(x)$ is bounded continuous on $\mathbb{R}_{+}$and it holds

$$
\sup _{x>0}|(G g)(x)| \leq \sqrt{\pi}|| g \|_{L_{1}(\mathbb{R})}
$$

Moreover, if $(G g)(x) \in L_{v, 1}\left(\mathbb{R}_{+}\right), 0<v<1 / 2$, then for all $y>0$

$$
\frac{1}{2 \pi i} \int_{v-i \infty}^{v+i \infty} \Gamma(s) \Gamma^{2}(1-s)(G g)^{*}(s) y^{-s} d s=\sqrt{\pi} \int_{-\infty}^{\infty} e^{y / 2} K_{i \tau}\left(\frac{y}{2}\right) \frac{g(\tau)}{\cosh (\pi \tau)} d \tau .
$$

Proof. First we observe from the familiar Poisson integral for the Bessel function [2], Vol. II that for all $x>0$

Therefore,

$$
\left|J_{i \tau}(\sqrt{x})\right| \leq \frac{\sqrt{\pi}}{|\Gamma(i \tau+1 / 2)|} .
$$

$$
\frac{\left|\operatorname{Re}\left[J_{i \tau}^{2}(\sqrt{x})\right]\right|}{\cosh (\pi \tau)} \leq 1
$$

and we find the estimate

$$
|(G g)(x)| \leq \sqrt{\pi} \int_{-\infty}^{\infty}|g(\tau)| d \tau=\left.\sqrt{\pi}|| g\right|_{L_{1}(\mathbb{R})},
$$


which yields (3.1). Next, integrating both sides of (1.2) with respect to $x$ and changing the order of integration under condition $g \in L_{1}(\mathbb{R})$, we get

$$
\int_{0}^{x}(G g)(y) d y=\int_{-\infty}^{\infty} \int_{0}^{x} \Phi_{\tau}(u) g(\tau) d u d \tau .
$$

Hence, employing (1.12), we derive

$$
\begin{gathered}
\frac{1}{x} \int_{0}^{x}(G g)(y) d y=\frac{1}{2 \pi i} \int_{-\infty}^{\infty} g(\tau) \int_{v-i \infty}^{v+i \infty} \frac{\Gamma(s+i \tau) \Gamma(s-i \tau) \Gamma(1 / 2-s)}{\Gamma(s) \Gamma(1-s) \Gamma(2-s)} x^{-s} d s d \tau \\
=\frac{1}{2 \pi i} \int_{v-i \infty}^{v+i \infty} \int_{-\infty}^{\infty} g(\tau) \frac{\Gamma(s+i \tau) \Gamma(s-i \tau) \Gamma(1 / 2-s)}{\Gamma(s) \Gamma(1-s) \Gamma(2-s)} x^{-s} d \tau d s,
\end{gathered}
$$

where the interchange of the order of integration is motivated by the straightforward estimate

$$
\begin{gathered}
\int_{v-i \infty}^{v+i \infty} \int_{-\infty}^{\infty}|g(\tau)| \frac{\Gamma(s+i \tau) \Gamma(s-i \tau) \Gamma(1 / 2-s)}{\Gamma(s) \Gamma(1-s) \Gamma(2-s)} x^{1-s}|d \tau| d s \mid \\
\leq x^{1-v} B(v, v) \int_{-\infty}^{\infty}|g(\tau)| d \tau \int_{v-i \infty}^{v+i \infty}\left|\frac{\Gamma(2 s) \Gamma(1 / 2-s)}{\Gamma(s) \Gamma(1-s) \Gamma(2-s)}\right||d s|<\infty, 0<v<\frac{1}{2}
\end{gathered}
$$

and $B(a, b)$ is Euler's beta-function [2], Vol. I. But under the condition $(G g)(x) \in L_{v, 1}\left(\mathbb{R}_{+}\right)$the Mellin transform (2.1) of the left-hand side in (3.4) exists and we have

$$
\int_{0}^{\infty} x^{s-2} \int_{0}^{x}(G g)(y) d y d x=\int_{0}^{\infty}(G g)(y) \int_{y}^{\infty} x^{s-2} d x d y=\frac{(G g)^{*}(s)}{1-s}, \operatorname{Re} s=v \in\left(0, \frac{1}{2}\right) .
$$

Thus (3.4) and the reduction formula for the gamma-function imply

$$
\Gamma(s) \Gamma^{2}(1-s)(G g)^{*}(s)=\Gamma(1 / 2-s) \int_{-\infty}^{\infty} g(\tau) \Gamma(s+i \tau) \Gamma(s-i \tau) d \tau
$$

Hence the inverse Mellin transform (2.3) and relation (8.4.23.5) in [4], Vol. III will lead us to (3.2), completing the proof of Theorem 3 .

The inversion formula for the index transform (1.2) is given by

Theorem 4. Let $g(z / i)$ be an even analytic function in the strip $D=\{z \in \mathbb{C}:|\operatorname{Re} z|<\alpha<1 / 2\}, g(0)=$ $g^{\prime}(0)=0$ and $g(z / i)$ is absolutely integrable over any vertical line in $D$. If $(G g)(t) \in L_{1}\left((0,1) ; t^{-1} d t\right) \cap$ $L_{1}((1, \infty) ; d t)$, then for all $x \in \mathbb{R}$ the inversion formula holds for the index transform (1.2)

$$
\begin{aligned}
& g(x)=\int_{0}^{\infty}(G g)(y)\left[\frac{\cosh (\pi x)}{\pi \sqrt{\pi}}+\pi x y \operatorname{coth}(\pi x) \frac{d}{d y}\left[\frac{\operatorname{Im}\left[J_{i x}^{2}(\sqrt{y})\right]}{\sinh (\pi x)}\right.\right. \\
& \left.\left.-\frac{1}{\sqrt{\pi}}\left[\left.J_{i x}(\sqrt{y}) \frac{\partial J_{\mathcal{E}-i x}(\sqrt{y})}{\partial \varepsilon}\right|_{\varepsilon=0}+\left.J_{-i x}(\sqrt{y}) \frac{\partial J_{\varepsilon+i x}(\sqrt{y})}{\partial \varepsilon}\right|_{\varepsilon=0}\right]\right]\right] \frac{d y}{y} .
\end{aligned}
$$

Proof. Indeed, recalling (3.2), we multiply its both sides by $e^{-y / 2} K_{i x}(y / 2) y^{\varepsilon-1}$ for some positive $\varepsilon \in(0,1)$ and integrate with respect to $y$ over $(0, \infty)$. Hence changing the order of integration in the left-hand side of the obtained equality due to the absolute convergence of the iterated integral, we appeal to relation (8.4.23.3) in [4], Vol. III to find

$$
\begin{gathered}
\frac{1}{2 \pi i} \int_{v-i \infty}^{v+i \infty} \frac{\Gamma(\varepsilon-s+i x) \Gamma(\varepsilon-s-i x)}{\Gamma(1 / 2+\varepsilon-s)} \Gamma(s) \Gamma^{2}(1-s)(G g)^{*}(s) d s \\
=\int_{0}^{\infty} K_{i x}\left(\frac{y}{2}\right) y^{\varepsilon-1} \int_{-\infty}^{\infty} K_{i \tau}\left(\frac{y}{2}\right) \frac{g(\tau)}{\cosh (\pi \tau)} d \tau d y .
\end{gathered}
$$


Meanwhile, the right-hand side of (3.6) can be treated in the same manner as in the proof of Theorem 4 in [7]. In fact, the evenness of $g$, a representation of the Macdonald function in terms of the modified Bessel function of the first kind $I_{z}(y)$ [2], Vol. II and a simple substitution give the equality

$$
\begin{aligned}
& \int_{0}^{\infty} K_{i x}\left(\frac{y}{2}\right) y^{\varepsilon-1} \int_{-\infty}^{\infty} K_{i \tau}\left(\frac{y}{2}\right) \frac{g(\tau)}{\cosh (\pi \tau)} d \tau d y \\
= & 2 \pi i \int_{0}^{\infty} K_{i x}\left(\frac{y}{2}\right) y^{\varepsilon-1} \int_{-i \infty}^{i \infty} I_{z}\left(\frac{y}{2}\right) \frac{g(z / i)}{\sin (2 \pi z)} d z d y .
\end{aligned}
$$

On the other hand, according to our assumption $g(z / i)$ is analytic in the vertical strip $0 \leq \operatorname{Re} z<\alpha<1 / 2$, $g(0)=g^{\prime}(0)=0$ and integrable in the strip. Hence, appealing to the inequality for the modified Bessel function of the first kind (see [3], p. 93)

$$
\left|I_{z}(y)\right| \leq I_{\operatorname{Re} z}(y) e^{\pi|\operatorname{Im} z| / 2}, 0<\operatorname{Re} z<\alpha,
$$

one can move the contour to the right in the latter integral in (3.7). Then

$$
\begin{aligned}
& 2 \pi i \int_{0}^{\infty} K_{i x}\left(\frac{y}{2}\right) y^{\varepsilon-1} \int_{-i \infty}^{i \infty} I_{z}\left(\frac{y}{2}\right) \frac{g(z / i)}{\sin (2 \pi z)} d z d y \\
= & 2 \pi i \int_{0}^{\infty} K_{i x}\left(\frac{y}{2}\right) y^{\varepsilon-1} \int_{\alpha-i \infty}^{\alpha+i \infty} I_{z}\left(\frac{y}{2}\right) \frac{g(z / i)}{\sin (2 \pi z)} d z d y .
\end{aligned}
$$

Now $\operatorname{Re} z>0$, and it is possible to pass to the limit under the integral sign when $\varepsilon \rightarrow 0$ and to change the order of integration due to the absolute and uniform convergence. Therefore the value of the integral (see relation (2.16.28.3) in [4], Vol. II)

leads us to the equalities

$$
\int_{0}^{\infty} K_{i x}(y) I_{z}(y) \frac{d y}{y}=\frac{1}{x^{2}+z^{2}}
$$

$$
\begin{gathered}
\lim _{\varepsilon \rightarrow 0} 2 \pi i \int_{0}^{\infty} K_{i x}\left(\frac{y}{2}\right) y^{\varepsilon-1} \int_{-i \infty}^{i \infty} I_{z}\left(\frac{y}{2}\right) \frac{g(z / i)}{\sin (2 \pi z)} d z d y \\
=2 \pi i \int_{\alpha-i \infty}^{\alpha+i \infty} \frac{g(z / i)}{\left(x^{2}+z^{2}\right) \sin (2 \pi z)} d z=\pi i\left(\int_{-\alpha+i \infty}^{-\alpha-i \infty}+\int_{\alpha-i \infty}^{\alpha+i \infty}\right) \frac{g(z / i) d z}{(z-i x) z \sin (2 \pi z)} .
\end{gathered}
$$

Hence conditions of the theorem allow to apply the Cauchy formula in the right-hand side of the latter equality in (3.8). Thus

$$
\lim _{\varepsilon \rightarrow 0} 2 \pi i \int_{0}^{\infty} K_{i x}\left(\frac{y}{2}\right) y^{\varepsilon-1} \int_{-i \infty}^{i \infty} I_{z}\left(\frac{y}{2}\right) \frac{g(z / i)}{\sin (2 \pi z)} d z d y=\frac{2 \pi^{2} g(x)}{x \sinh (2 \pi x)}, \quad x \in \mathbb{R} \backslash\{0\} .
$$

Now, recalling the Parseval identity (2.2), the left-hand side of (3.6) can be rewritten in the form

$$
\frac{1}{2 \pi i} \int_{v-i \infty}^{v+i \infty} \frac{\Gamma(\varepsilon-s+i x) \Gamma(\varepsilon-s-i x)}{\Gamma(1 / 2+\varepsilon-s)} \Gamma(s) \Gamma^{2}(1-s)(G g)^{*}(s) d s=\int_{0}^{\infty}(G g)(y) \Psi_{\varepsilon}(x, y) \frac{d y}{y},
$$

where

$$
\Psi_{\varepsilon}(x, y)=\frac{1}{2 \pi i} \int_{1-v-i \infty}^{1-v+i \infty} \frac{\Gamma(s+\varepsilon-1+i x) \Gamma(s+\varepsilon-1-i x)}{\Gamma(s+\varepsilon-1 / 2)} \Gamma^{2}(s) \Gamma(1-s) y^{1-s} d s .
$$

Meanwhile, relations (8.4.23.3), (8.4.11.3) in [4], Vol. III and the Parseval equality (2.2) give the representation (3.11) accordingly

$$
\Psi_{\varepsilon}(x, y)=\frac{y^{\varepsilon}}{\sqrt{\pi}} \int_{0}^{\infty} \int_{1}^{\infty} \exp \left(u(1-t)-\frac{y}{2 u}\right) K_{i x}\left(\frac{y}{2 u}\right) \frac{d t d u}{t u^{\varepsilon}}
$$




$$
\begin{gathered}
=\frac{y^{\varepsilon}}{\sqrt{\pi}} \int_{0}^{\infty} \frac{d t}{1+t} \int_{0}^{\infty} \exp \left(-\frac{t}{u}-\frac{y u}{2}\right) K_{i x}\left(\frac{y u}{2}\right) \frac{d u}{u^{2-\varepsilon}} \\
=-\frac{y^{\varepsilon}}{\sqrt{\pi}} \int_{0}^{\infty} \frac{d t}{1+t} \frac{d}{d t} \int_{0}^{\infty} \exp \left(-\frac{t}{u}-\frac{y u}{2}\right) K_{i x}\left(\frac{y u}{2}\right) u^{\varepsilon-1} d u, \varepsilon>0,
\end{gathered}
$$

where the interchange of the order of integration and differentiation under the integral sign are permitted owing to the absolute and uniform convergence. Integrating by parts in the latter integral with respect to $t$ and using relation (2.16.9.3) in [4], Vol. II, we find

$$
\Psi_{\varepsilon}(x, y)=\frac{|\Gamma(\varepsilon+i x)|^{2}}{\Gamma(\varepsilon+1 / 2)}-\frac{y^{\varepsilon}}{\sqrt{\pi}} \int_{0}^{\infty} \frac{d t}{(1+t)^{2}} \int_{0}^{\infty} \exp \left(-\frac{t}{u}-\frac{y u}{2}\right) K_{i x}\left(\frac{y u}{2}\right) u^{\varepsilon-1} d u .
$$

Moreover, it is possible now to pass to the limit under the integral sign in (3.12) when $\varepsilon \rightarrow 0$ and then to employ relation (2.16.9.1) in [4], Vol. II. Hence, reminding (2.17), we obtain

$$
\Theta(x, y)=\lim _{\varepsilon \rightarrow 0} \Psi_{\varepsilon}(x, y)=\frac{\sqrt{\pi}}{x \sinh (\pi x)}-\frac{2}{\sqrt{\pi}} \int_{0}^{\infty} \frac{K_{i x}^{2}(\sqrt{y t}) d t}{(1+t)^{2}}=\frac{\sqrt{\pi}}{x \sinh (\pi x)}+2 y K(y, x) .
$$

In the meantime, passing to the limit in (3.10) when $\varepsilon \rightarrow 0$, we do it under the integral sign in its right-hand side for each $x \in \mathbb{R} \backslash\{0\}$, appealing to the dominated convergence theorem due to the condition $(G g)(x) \in$ $L_{1}\left((0,1) ; x^{-1} d x\right) \cap L_{1}((1, \infty) ; d x)$ and the absolute and uniform convergence with respect to $\varepsilon \in[0,1]$. In fact, since from (3.12)

$$
\begin{gathered}
\left|\Psi_{\varepsilon}(x, y)\right| \leq \frac{|\Gamma(\varepsilon+i x)|^{2}}{\Gamma(\varepsilon+1 / 2)}+\frac{y^{\varepsilon}}{\sqrt{\pi}} \int_{0}^{\infty} \frac{d t}{(1+t)^{2}} \int_{0}^{1} \exp \left(-\frac{t}{u}-\frac{y u}{2}\right) K_{0}\left(\frac{y u}{2}\right) \frac{d u}{u} \\
+\frac{y^{\varepsilon}}{\sqrt{\pi}} \int_{0}^{\infty} \frac{d t}{(1+t)^{2}} \int_{1}^{\infty} \exp \left(-\frac{t}{u}-\frac{y u}{2}\right) K_{0}\left(\frac{y u}{2}\right) d u \\
\leq \frac{|\Gamma(\varepsilon+i x)|^{2}}{\Gamma(\varepsilon+1 / 2)}+\frac{2 y^{\varepsilon}}{\sqrt{\pi}} \int_{0}^{\infty} \frac{K_{0}^{2}(\sqrt{y t}) d t}{(1+t)^{2}} \\
+\frac{y^{\varepsilon-1}}{\sqrt{\pi}} \int_{0}^{\infty} \frac{d t}{(1+t)^{2}} \int_{0}^{\infty} \exp \left(-\frac{u}{2}\right) K_{0}\left(\frac{u}{2}\right) d u \\
=O(1)+O\left(y^{\varepsilon-1}\right),
\end{gathered}
$$

we have

$$
\int_{0}^{\infty}\left|(G g)(y) \Psi_{\varepsilon}(x, y)\right| \frac{d y}{y} \leq C_{1} \int_{0}^{1}|(G g)(y)| \frac{d y}{y}+C_{2} \int_{1}^{\infty}|(G g)(y)| d y<\infty,
$$

where $C_{1}, C_{2}>0$ are absolute constants. Hence, combining with (3.9) and (2.21) we arrive at the inversion formula (3.5). Theorem 4 is proved.

\section{INITIAL VALUE PROBLEM}

In this section we will apply the index transform (1.2) to investigate the solvability of an initial value problem for the following third order partial differential equation, involving the Laplacian

$$
\left(x \frac{\partial}{\partial x}+y \frac{\partial}{\partial y}+2\right) \Delta u+\frac{\sqrt{x^{2}+y^{2}}-8}{x^{2}+y^{2}}\left[x \frac{\partial u}{\partial x}+y \frac{\partial u}{\partial y}\right]+\frac{u}{2 \sqrt{x^{2}+y^{2}}}=0,(x, y) \in \mathbb{R}^{2} \backslash\{0\},
$$


where $\Delta=\frac{\partial^{2}}{\partial x^{2}}+\frac{\partial^{2}}{\partial y^{2}}$ is the Laplacian in $\mathbb{R}^{2}$. In fact, writing (4.1) in polar coordinates $(r, \theta)$, we end up with the equation

$$
r \frac{\partial^{3} u}{\partial r^{3}}+\frac{1}{r} \frac{\partial^{3} u}{\partial r \partial^{2} \theta}+3 \frac{\partial^{2} u}{\partial r^{2}}+\left[1-\frac{7}{r}\right] \frac{\partial u}{\partial r}+\frac{u}{2 r}=0 .
$$

Lemma 3. Let $g(\tau) \in L_{1}\left(\mathbb{R} ; e^{\beta|\tau|} d \tau\right), \beta \in(0,2 \pi)$. Then the function

$$
u(r, \theta)=\sqrt{\pi} \int_{-\infty}^{\infty} \operatorname{Re}\left[J_{i \tau}^{2}(\sqrt{r})\right] \frac{e^{\theta \tau} g(\tau) d \tau}{\cosh (\pi \tau)}
$$

satisfies the partial differential equation (4.2) on the wedge $(r, \theta): r>0,0 \leq \theta<\beta$, vanishing at infinity.

Proof. The proof is straightforward by substitution (4.3) into (4.2) and the use of (1.15). The necessary differentiation with respect to $r$ and $\theta$ under the integral sign is allowed via the absolute and uniform convergence, which can be verified using inequality (3.3) and the integrability condition $g \in L_{1}\left(\mathbb{R} ; e^{\beta|\tau|} d \tau\right), \beta \in$ $(0,2 \pi)$ of the lemma. Finally, the condition $u(r, \theta) \rightarrow 0, r \rightarrow \infty$ is due to the asymptotic formula (1.4) for Bessel function at infinity.

Finally we will formulate the initial value problem for equation (4.2) and give its solution.

Theorem 5. Let $g(x)$ be given by formula (3.5) and its transform $(G g)(t) \equiv G(t)$ satisfies conditions of Theorem 4. Then $u(r, \theta), r>0,0 \leq \theta<\beta$ by formula (4.3) will be a solution of the initial value problem for the partial differential equation (4.2) subject to the initial condition

$$
u(r, 0)=G(r) .
$$

\section{Acknowledgments}

The work was partially supported by CMUP (UID/MAT/00144/2013), which is funded by FCT(Portugal) with national (MEC) and European structural funds through the programs FEDER, under the partnership agreement PT2020.

\section{REFERENCES}

1. Yakubovich S. Index transforms. Singapore: World Scientific Publishing Company; 1996.

2. Erdélyi A, Magnus W, Oberhettinger F, Tricomi FG. Higher transcendental functions. Vols. I, II. New York: McGraw-Hill; 1953.

3. Yakubovich S, Luchko Yu. The hypergeometric approach to integral transforms and convolutions, Mathematics and its applications. Vol. 287. Dordrecht: Kluwer Academic Publishers Group; 1994.

4. Prudnikov AP, Brychkov YuA, Marichev OI. Integrals and series: Vol. I: Elementary functions. New York: Gordon and Breach; 1986; Vol. II: Special functions. New York: Gordon and Breach; 1986; Vol. III: More special functions. New York: Gordon and Breach; 1990.

5. Titchmarsh EC. An introduction to the theory of Fourier integrals. New York: Chelsea; 1986.

6. Lebedev NN. On an integral representation of an arbitrary function in terms of squares of Macdonald functions with imaginary index, Sibirsk. Mat. Zh., 3 (1962), 213- 222 (in Russian).

7. Yakubovich S. New index transforms of the Lebedev- Skalskaya type, arXiv: 1509.02764.

Department of Mathematics, Faculty of Sciences, University of Porto, Campo Alegre str., 687; 4169 -007 PORTO, PORTUGAL

E-mail address: $\quad$ syakubov@fc.up.pt 